\section{Variáveis associadas ao câncer de mama em usuárias de unidades básicas de saúde}

\author{
Variables associated with breast cancer in \\ clients of primary healthcare units
}

\author{
${ }^{1}$ Centro Nacional de \\ Transplante de Medula \\ Óssea, Instituto Nacional de \\ Câncer, Rio de Janeiro, Brasil. \\ 2 Escola Nacional de Saúde \\ Pública Sergio Arouca, \\ Fundação Oswaldo Cruz, \\ Rio de Janeiro, Brasil. \\ Correspondência \\ V. F. S. Pinho \\ Centro Nacional de \\ Transplante de Medula \\ Óssea, Instituto Nacional \\ de Câncer. \\ Praça da Cruz Vermelha 23 \\ Rio de Janeiro, $R J$ \\ 20230-130, Brasil. \\ vdesouzapinho@yahoo.com.br
}

\begin{abstract}
An epidemiological survey in ten family health units in the municipality of Teresópolis, Rio de Janeiro State, Brazil, in 2003, investigated the distribution pattern of breast cancer risk factors. 698 women 25 to 88 years of age were interviewed (112 rural and 586 urban). As for factors associated with breast cancer, $36.1 \%$ were 50 years or older, $3.7 \%$ had a first-degree family history, and $0.4 \%$ had a prior diagnosis of breast cancer. As for other factors, prevalence was higher in women with a history of abortion (38.5\%), breastfeeding of less than one year (37.4\%), prolonged use of oral contraceptives (41.1\%), low physical activity (58.7\%), and obesity (30\%). Although the latter factors are still under investigation and the measures found in studies show weak associations, they are relevant to public health, not only for breast cancer control but also for other diseases, since their prevalence is high in the population.
\end{abstract}

Risk Factors; Breast Neoplasms; Women's Health
Valéria Fernandes de Souza Pinho 1 Evandro Silva Freire Coutinho 2

\section{Introdução}

O câncer de mama é o tumor invasivo que mais acomete e mata as mulheres no Brasil. Entre 1980 e 2000, a mortalidade bruta para o câncer de mama passou de 6,14 para 9,64 por 100 mil mulheres, resultando em um aumento relativo de $57 \%$ (DATASUS. Informações de saúde. http://www. datasus.gov.br, acessado em Abr/2004). Embora essa variação possa refletir também uma melhora das técnicas de diagnóstico e do registro nas declarações de óbitos, sua principal causa é que nossas medidas de rastreamento e controle da doença ainda são muito deficientes. Cerca de $60 \%$ dos nossos casos são diagnosticados em estágios avançados, ainda não se dispõe de uma estrutura que garanta a mamografia sistemática a todas as mulheres nas faixas etárias de maior risco e a rede de assistência oncológica no país é insuficiente, inadequada e mal distribuída 1,2,3. Por outro lado, em países desenvolvidos, como Estados Unidos, Canadá, Reino Unido, Holanda, Dinamarca e Noruega, a incidência da doença é alta e vem aumentando a cada ano, mas a mortalidade vem diminuindo graças à detecção precoce pela mamografia e pelo tratamento adequado dos casos 4,5 .

Diante dessa situação, o Ministério da Saúde, por intermédio do Instituto Nacional de Câncer (INCA) e juntamente com outras organizações, vem traçando diretrizes para o enfrentamento desse problema, com a implantação e desen- 
volvimento do Programa Nacional de Controle do Câncer do Colo de Útero e de Mama - Viva Mulher. O programa tem por objetivo reduzir a mortalidade e as repercussões físicas, sociais e psíquicas da doença, com a oferta de serviços de prevenção, detecção precoce, tratamento e reabilitação 6 .

A pesquisa de variáveis associadas para o câncer de mama pode estar relacionada aos programas de controle da doença. De acordo com estudos bem desenhados e controlados, a idade elevada, geralmente após os 50 anos, é o fator mais importante, e, na maioria dos casos, é o único encontrado. Contudo, outros fatores também já estão estabelecidos na causalidade da doença, como história familiar e pessoal de câncer de mama; antecedente pessoal de hiperplasia atípica ou carcinoma lobular in situ; menarca precoce; menopausa tardia; nuliparidade; primeira gestação após 30 anos; terapia de reposição hormonal; uso prolongado de contraceptivos orais; exposição a altas doses de radiação ionizante e obesidade na pós-menopausa. Além desses, outros fatores também estão sendo investigados, mas os resultados dos estudos ainda não são conclusivos. São eles: sedentarismo, consumo de álcool e gorduras, história de abortos, não-amamentação, tabagismo e exposição a toxinas ambientais $4,7,8,9$.

Embora a idade seja o único critério de seleção para programas padronizados de rastreamento, a identificação de outras variáveis associadas, em diferentes grupos populacionais, permite não só aumentar o conhecimento sobre a epidemiologia dessa neoplasia, como também identificar mulheres que poderiam se beneficiar com um rastreamento diferenciado 10,11. Por exemplo, para a detecção precoce da doença, o Programa Viva Mulher tem como população-alvo as usuárias da rede básica do Sistema Único de Saúde (SUS). As ações são feitas por meio do exame clínico anual das mamas, do ensino da técnica de auto-exame destas e da educação para a saúde em mulheres na faixa etária de 25 a 69 anos ${ }^{6}$. Em relação à mamografia, o último consenso entre especialistas no assunto e organizações afins recomenda que as mulheres de alto risco com 35 anos ou mais, ou seja, aquelas que apresentam fatores de forte associação com o câncer de mama, devam ser acompanhadas anualmente com um exame clínico e uma radiografia, mesmo sem sinais ou sintomas. Recomenda, ainda, que todas as mulheres na faixa etária de 50 a 69 anos e que estejam assintomáticas façam uma mamografia pelo menos a cada dois anos 5 .

O programa classifica como de alto risco as mulheres que tenham antecedente familiar de câncer de mama em parentes diretos (mãe, irmã ou filha), ocorrido antes dos 50 anos, e/ou antecedente pessoal de hiperplasia atípica ou carcinoma lobular in situ ${ }^{6}$. O consenso supracitado também incluiu neste grupo as mulheres com antecedente familiar de câncer de mama bilateral, câncer de mama masculino e/ou câncer de ovário, em qualquer faixa etária 5 .

No Brasil, alguns estudos sobre variáveis associadas para o câncer de mama foram desenvolvidos, contudo a maioria deles foi realizada com base em registros de instituições de tratamento ou de diagnóstico, sendo pesquisas de associação nas quais nem sempre é possível estimar a prevalência de um fator de risco de modo não viesado 12 . Portanto, pouco se conhece sobre a prevalência desses fatores na população geral, e as usuárias de unidades básicas de saúde, que são os alvos dos programas de detecção precoce, foram pouco investigadas nesse aspecto.

Em face dessas considerações, nosso objetivo foi estimar o padrão de distribuição das variáveis associadas para o câncer de mama em usuárias de tais unidades, comparando os achados entre os estratos urbano e rural dessa população.

\section{Métodos}

No período de junho a novembro de 2003, foi realizado um inquérito epidemiológico no Município de Teresópolis, situado na Região Serrana do Estado do Rio de Janeiro, Brasil, que possui 138.081 habitantes, dos quais $70.970(51,4 \%)$ são mulheres e 22.883 (16,6\%) residem na área rural (Instituto Brasileiro de Geografia e Estatística; http://www.ibge.gov.br acessado em Set/2003). A população-alvo foi constituída de mulheres cadastradas no Programa Saúde da Família (PSF) do município, que cobre em torno de $29 \%$ dos residentes e possui um total de dez unidades, duas localizadas na área rural e oito na área urbana da cidade 13 .

Com base nesses dados, estimou-se que 12.120 mulheres na faixa etária de 25 anos ou mais estariam cadastradas no programa. As unidades do PSF foram escolhidas pela importância que vêm assumindo na assistência à saúde da mulher; além disso, trata-se de um programa que tem melhor controle da sua população através de um cadastro de famílias assistidas e do acompanhamento destas pelos agentes comunitários de saúde.

O cálculo da amostra foi feito com base numa prevalência esperada de $2,1 \%$ para o grau de alto risco para o câncer de mama (estimada no estudo transversal de Lima \& Falk 14, realizado com mulheres nordestinas atendidas na triagem de um hospital de referência para o tratamento do 
câncer em Recife, Pernambuco) e num erro bilateral tolerável de 1,1\% para um nível de confiança de $95 \%$. Dessa forma, obtivemos um resultado de 620 mulheres. Esse quantitativo foi distribuído proporcionalmente entre as unidades, de acordo com o número de mulheres cadastradas em cada uma delas (dados fornecidos em março de 2003 pela coordenação do PSF no município).

A seleção da amostra foi sistemática e baseou-se na dinâmica de atendimento das unidades, que obedecia ao agendamento prévio de consultas e procedimentos, feito por ordem de chegada. Os critérios de seleção foram os seguintes: ter 25 anos ou mais e estar cadastrada no PSF. A meta foi avaliar todas as mulheres elegíveis que estivessem presentes nos turnos de trabalho das Unidades de Saúde da Família (USF), manhã e tarde, não só as que estavam agendadas para atendimento próprio, como também as que acompanhavam familiares agendados.

Os dados foram obtidos mediante entrevista estruturada, utilizando-se um questionário composto de questões simples com respostas objetivas e curtas. Esse instrumento foi construído com base no questionário utilizado por Mendonça 15, no Breast Cancer Risk Assessment Tool (National Cancer Institute; http:// www.brca.nci. nih.gov, acessado em Mai/2002) e no Harvard Cancer Risk Index 16. Além desses, por recomendação de um especialista em nutrição de que se avaliasse a ingestão de alimentos gordurosos, foi utilizado o questionário simplificado de consumo de alimentos de risco para doença cardiovascular, desenvolvido por Chiara \& Sichieri 17, testado e validado em adolescentes da cidade do Rio de Janeiro. Este instrumento considera os seguintes alimentos: batata frita, carne vermelha, biscoitos, bolos ou tortas, leite integral, hambúrguer, queijo, manteiga ou margarina e salsicha. O resultado é quantitativo, obtido com o somatório da pontuação atribuída à freqüência de consumo de cada alimento na última semana, e são especificados os seguintes pontos de corte: até 100 pontos - consumo adequado; de 101 a 119 pontos - consumo alto e 120 pontos ou mais - consumo excessivo de alimentos gordurosos.

Embora os instrumentos que subsidiaram o questionário já tivessem sido validados em outras populações, ele foi previamente administrado a um grupo de 100 mulheres como teste-piloto para avaliação da clareza e objetividade das questões, bem como da facilidade das respostas e duração da entrevista.

As variáveis pesquisadas foram características sócio-demográficas, história hormonal e reprodutiva, hábitos de vida, história familiar, história pregressa e dados antropométricos, cujas categorias assinaladas com asteriscos são aquelas que se acredita associadas com um aumento do risco para o câncer de mama:

- Idade (50 anos ou mais*; menos de 50 anos).

- Menarca (antes dos 12 anos*; 12 anos ou mais).

- Nuliparidade (sim*; não).

- História de um ou mais abortos espontâneos e/ou provocados (sim*; não).

- Idade na primeira gestação a termo (após os 30 anos*; até 30 anos).

- Idade na última gestação a termo (após 30 anos*; até 30 anos).

- Não amamentação ou amamentação total por menos de um ano (sim*; não): somatório dos períodos de amamentação de todos os filhos.

- Uso de contraceptivos orais (cinco anos ou mais*; menos de cinco anos).

- Uso de terapia de reposição hormonal (cinco anos ou mais*; menos de cinco anos).

- Idade da menopausa (55 anos ou mais*; até 54 anos).

- Sedentarismo (ausência de atividade física regular pelo menos três vezes na semana*; três vezes ou mais por semana).

- Ingestão de bebidas alcoólicas (consumo de mais de uma lata de cerveja, mais de um copo de vinho e/ou mais de uma dose de bebidas destiladas por dia*; menos que essa quantidade por dia).

- Tabagismo atual (sim*; não).

- Ingestão excessiva de alimentos gordurosos (120 ou mais pontos na aplicação do questionário de consumo alimentar descrito acima*, menos de 120 pontos).

- Estatura (1,70 metros ou mais*; menos de 1,70 metros).

- Obesidade (índice de massa corporal - IMC de 30,00 ou mais*; IMC abaixo de 30,00).

- Antecedente familiar de câncer de mama em qualquer grau (sim*, não).

- Antecedente pessoal de biópsia de mama cujo resultado tenha sido hiperplasia atípica ou carcinoma lobular in situ (sim*, não).

- Antecedente pessoal de câncer de mama (sim*, não).

O entrevistador (pesquisador) permanecia na unidade até o encerramento dos turnos, quantos dias fossem necessários para completar o número de entrevistas estipuladas para cada USF. Com exceção da unidade onde foi feito o teste-piloto, a seqüência de trabalho deu-se por sorteio. As mulheres eram abordadas enquanto esperavam o atendimento e recebiam as devidas informações sobre a pesquisa e seus objetivos, juntamente com a garantia de confidencialidade dos dados. Após o consentimento assinado, o entrevistador procedia à entrevista e, depois desta, aferia 
o peso e altura das entrevistadas em balanças de adulto padronizadas, em todas as unidades.

As informações provenientes dos questionários foram categorizadas, codificadas e digitadas em um banco de dados confeccionado no programa estatístico SPSS 9.0 (SPSS Inc., Chicago, Estados Unidos), no qual foram calculadas as freqüências absolutas e proporções das variáveis pesquisadas, bem como a média e o desvio-padrão de variáveis contínuas. Para a avaliação da precisão das proporções, foi calculado o intervalo de confiança de 95\% através do módulo "Epitable” do Epi Info 6.0 (Centers for Disease Control and Prevention, Atlanta, Estados Unidos); para comparação das proporções entre os estratos urbano e rural da amostra, foi realizado o teste quiquadrado no mesmo programa. As não-respostas foram codificadas como "inválidas" e não foram computadas na análise estatística. As questões sobre idade da menarca e idade ao nascimento do primeiro filho foram repetidas, após um período mínimo de dois dias, em uma subamostra de mulheres, e a confiabilidade desses dados foi estimada por meio do coeficiente de correlação intraclasse.

A Secretaria Municipal de Saúde de Teresópolis autorizou a coleta de dados nas USF e o projeto deste estudo foi aprovado pelo Comitê de Ética da Escola Nacional de Saúde Pública Sergio Arouca, Fundação Oswaldo Cruz. O consentimento das mulheres para participar da pesquisa foi escrito e assinado; aquelas que não sabiam assinar o nome fizeram a impressão dactiloscópica de seu polegar direito. A proteção da identidade das participantes foi mantida durante todo o processo de coleta dos dados e divulgação dos resultados.

\section{Resultados}

Foram entrevistadas 698 mulheres, sendo 112 provenientes da área rural e 586 da área urbana da cidade. O tempo médio de duração das entrevistas foi de dez minutos, com uma variação de 5 a 34 minutos. Oito mulheres ( $1 \%$ da amostra) recusaram-se a participar do estudo; outras nove $(1,3 \%)$, em virtude de o fluxo das consultas, em alguns dias, ter sido mais rápido do que o das entrevistas, deixaram as unidades antes de serem entrevistadas e não foi possível recuperá-las.

\section{Características sócio-demográficas}

Oitenta e quatro por cento das mulheres entrevistadas residiam na área urbana. A idade delas variou de 25 a 88 anos, com uma média de 45,5 $(\mathrm{DP}=15)$. Na comparação entre os estratos, ob- servou-se maior prevalência de mulheres com 50 anos ou mais no grupo urbano. Mais de $80 \%$ delas não completaram a escolaridade fundamental, e um quarto destas era de analfabetas, principalmente rurais. Quanto à situação conjugal, menos de $10 \%$ eram solteiras (sem companheiro estável).

Mais da metade das entrevistadas era dona de casa ou estava aposentada, principalmente na área urbana. Das mulheres urbanas que trabalhavam, mais de $50 \%$ eram empregadas domésticas. Já na área rural, em 31\% dos casos, a principal ocupação foi a agricultura. Em relação à renda mensal, mais de $30 \%$ das mulheres não souberam prestar esta informação (ou por a renda ser proveniente de trabalho informal ou por desconhecerem o salário do companheiro), com maior prevalência de não-respostas no meio rural. Das que o fizeram, a grande maioria referiu menos de um salário mínimo per capita. O tamanho das famílias variou de 1 a 12 pessoas, com uma média de $4(\mathrm{DP}=2)$. O grupo rural foi composto em sua maioria de acompanhantes, ao passo que, do grupo urbano, $72 \%$ foram à unidade para atendimento próprio.

\section{Variáveis associadas para o câncer de mama}

A maior parte dos resultados sobre variáveis associadas para o câncer de mama está apresentada na Tabela 1. Outros achados são descritos a seguir: quase $50 \%$ das entrevistadas tiveram o primeiro filho antes dos 20 anos de idade, e a média foi de quatro filhos ( $\mathrm{DP}=2,5)$.

Trinta por cento das entrevistadas eram menopausadas, com maior prevalência entre o meio urbano (37\% urbano x 18,7\% rural; p < 0,001). Cinqüenta e três (8\%) foram histerectomizadas, mas só foram consideradas menopausadas aquelas cuja cirurgia ocorreu após a idade média da menopausa no grupo estudado, que foi de 47 anos (DP = 7).

Dentre as mulheres que praticavam exercícios físicos, caminhada foi a atividade mais freqüente. A distribuição de consumo diário dos alimentos pesquisados foi a seguinte: manteiga ou margarina em $53 \%$ das mulheres; leite integral em 37\%; biscoitos doces ou salgados em $25 \%$; queijo em $10 \%$; carne vermelha em $6 \%$; bolos ou tortas em 1,5\%; batata frita em $0,9 \%$; salsicha em $0,6 \%$ e hambúrguer em $0 \%$. Pouco mais de um terço das mulheres tinha sobrepeso e $30 \%$ tinham obesidade (IMC $\geq 30,00$ ), mas sem diferenças entre os estratos.

Nenhuma das mulheres que apresentavam antecedente familiar de primeiro grau para o câncer de mama informou ter feito exames para a pesquisa de mutação genética de BRCA1/2. 
Freqüências de variáveis associadas para câncer de mama, estratos rural e urbano. Unidades de Saúde da Família, Teresópolis, Rio de Janeiro, Brasil, 2003.

\begin{tabular}{|c|c|c|c|c|c|c|c|}
\hline \multirow[t]{2}{*}{ Variável/Estrato } & \multicolumn{2}{|c|}{ Rural ( $n=112$ ) } & \multicolumn{2}{|c|}{ Urbano $(n=586)$} & \multicolumn{2}{|c|}{ Total $(n=698)$} & \multirow[t]{2}{*}{$p$ valor } \\
\hline & $\%$ & IC95\% & $\%$ & IC95\% & $\%$ & IC95\% & \\
\hline Idade $\geq 50$ anos & 25 & $17,3-34,1$ & 38,2 & $34,3-42,3$ & 36,1 & $32,5-39,8$ & 0,00 \\
\hline Menarca $<12$ anos & 29,1 & $20,6-38,9$ & 22,4 & $18,9-26,1$ & 23,4 & $20,2-26,9$ & 0,13 \\
\hline Nuliparidade & 4,5 & $1,5-10,1$ & 4,6 & $3,1-6,6$ & 4,6 & $3,2-6,4$ & 0,79 \\
\hline \multicolumn{8}{|l|}{ Abortos } \\
\hline 1 & 22,3 & $15,0-31,2$ & 23,1 & $19,4-27,2$ & 23,0 & $19,6-26,6$ & 0,86 \\
\hline$\geq 2$ & 9,8 & $5,0-16,9$ & 16,9 & $13,6-20,5$ & 15,5 & $12,7-18,7$ & 0,06 \\
\hline \multicolumn{8}{|l|}{ Primeira gestação (anos) } \\
\hline $30-33$ & 1,0 & $0,0-5,3$ & 3,0 & $1,7-4,8$ & 2,7 & $1,6-4,3$ & 0,40 * \\
\hline$\geq 34$ & 1,0 & $0,0-5,3$ & 2,3 & $1,7-3,9$ & 2,0 & $1,1-3,5$ & 0,64 * \\
\hline \multicolumn{8}{|l|}{ Última gestação (anos) } \\
\hline $30-33$ & 17,6 & $10,2-27,4$ & 23,3 & $19,4-27,5$ & 22,4 & $18,8-26,2$ & 0,25 \\
\hline$\geq 34$ & 30,6 & $21,0-41,5$ & 26,7 & $22,6-31,2$ & 27,4 & $23,6-31,4$ & 0,47 \\
\hline \multicolumn{8}{|l|}{ Amamentação } \\
\hline Nenhuma & 9,1 & $4,4-16,1$ & 13,0 & $10,4-16,0$ & 12,4 & $10,0-15,1$ & 0,25 \\
\hline$<1$ ano & 30,0 & $21,6-39,5$ & 29,1 & $26,1-33,7$ & 25,0 & $21,8-28,5$ & 0,85 \\
\hline Uso de contraceptivos orais por $\geq 5$ anos & 59,0 & $49,0-68,5$ & 37,8 & $33,7-41,9$ & 41,1 & $37,4-45,0$ & 0,00 \\
\hline Menopausa com $\geq 55$ anos & 2,8 & $0,6-8,0$ & 5,3 & $3,6-7,5$ & 4,4 & $3,0-6,3$ & 0,24 \\
\hline Uso de reposição hormonal por $\geq 5$ anos & 0,9 & $0,0-5,0$ & 3,0 & $1,7-4,7$ & 2,6 & $1,6-4,1$ & $0,37 *$ \\
\hline Sedentarismo & 48,2 & $38,7-57,9$ & 60,8 & $56,7-64,7$ & 58,7 & $55,0-62,4$ & 0,01 \\
\hline Consumo excessivo de bebidas alcoólicas & 0 & - & 0,9 & $0,3-2,0$ & 0,7 & $0,2-1,7$ & 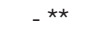 \\
\hline Tabagismo atual & 13,5 & $7,8-21,3$ & 17,2 & $14,2-20,5$ & 16,6 & $13,9-19,5$ & 0,34 \\
\hline Consumo excessivo de alimentos gordurosos & 6,3 & $2,5-12,5$ & 7,4 & $5,4-9,8$ & 7,2 & $5,4-9,4$ & 0,68 \\
\hline Estatura $\geq 1,70 m$ & 1,8 & $0,2-6,4$ & 0,7 & $0,2-1,7$ & 0,9 & $0,3-1,9$ & - \\
\hline Índice de massa corporal $\geq 30,00 \mathrm{Kg} / \mathrm{m}^{2}$ & 27,3 & $19,2-36,6$ & 30,5 & $26,7-34,5$ & 30,0 & $26,6-33,6$ & 0,49 \\
\hline \multicolumn{8}{|l|}{ Antecedente familiar de câncer de mama } \\
\hline 1으 grau & 1,8 & $0,2-6,4$ & 4,0 & $2,6-5,9$ & 3,7 & $2,4-5,4$ & 0,40 * \\
\hline 2o grau & 2,7 & $0,6-7,8$ & 7,1 & $5,2-9,6$ & 6,4 & $4,7-8,5$ & 0,08 \\
\hline Grau distante & 4,5 & $1,5-10,3$ & 1,4 & $0,6-2,7$ & 1,9 & $1,0-3,2$ & 0,07 * \\
\hline Antecedente pessoal de câncer de mama & 0 & - & 0,5 & $0,1-1,5$ & 0,4 & $0,0-1,3$ & - \\
\hline
\end{tabular}

Nota: A amostra nos estratos e no total variou em virtude de perdas de informações ou de dados não aplicáveis a determinadas categorias. O p valor foi obtido com a aplicação do teste qui-quadrado.

* Ajustado pela correção de Yates;

** Não foi possível realizar o teste qui-quadrado porque a freqüência das categorias nos estratos foi muito baixa.

Entre as 13 mulheres que foram submetidas a biópsias de mama, nenhuma referiu resultado de hiperplasia atípica ou carcinoma lobular in situ, embora o resultado do exame fosse desconhecido para três delas.

\section{Confiabilidade dos dados}

A confiabilidade dos dados foi testada em uma subamostra de 36 mulheres ( $5 \%$ da amostra); o coeficiente de correlação intraclasse para idade da menarca foi de 0,97 (IC95\%: 0,94-0,99) e para a idade ao nascimento do primeiro filho foi de 0,97 (IC95\%: 0,94-0,98).

\section{Discussão}

O câncer de mama é um dos tumores mais estudados no mundo inteiro, mas ainda existem muitas questões e controvérsias sobre os seus determinantes. A maioria dos estudos enfatiza a questão das variáveis associadas em grupos que já desenvolveram a doença, e muito pouco se sabe sobre a prevalência destes fatores em mulheres assintomáticas que formam a população-alvo de programas de detecção precoce.

Em seu conjunto, os dados deste estudo mostram um grupo composto em sua maioria de mulheres casadas, de baixa escolaridade e renda, pouco inseridas no mercado de trabalho e que freqüentaram as USF por problemas de saúde 
próprios ou de familiares. Alguns estudos mostraram que fatores sócio-demográficos podem estar associados a um maior risco de câncer de mama, mas a magnitude dessa associação é fraca, com riscos relativos inferiores a 2,0 9. Com exceção da idade, outras variáveis sócio-demográficas não devem ser utilizadas como parâmetros para rastreamento, por discriminarem pouco as portadoras da doença das mulheres saudáveis 11 .

Ao contrário dos fatores sócio-demográficos, as variáveis hormonais e reprodutivas são mais consideradas na etiologia do câncer de mama, e têm como plausibilidade biológica geral a exposição a estrógenos, progestágenos e outros hormônios de potencial carcinogênico 18,19,20. A menarca precoce esteve presente em quase um quarto da nossa amostra, mas essa prevalência é menor do que a encontrada no estudo de Lamas \& Pereira ${ }^{11}$ em mulheres de Brasília, Distrito Federal (29,3\%).

A última gestação a termo após os 30 anos de idade teve alta prevalência (50\%). Embora a primeira gestação tardia seja a que já tem o seu papel estabelecido na causalidade do câncer de mama ? a idade na última gestação também está sendo investigada. Segundo o estudo caso-controle de Kalache et al. 21, realizado com mulheres de Recife, em Pernambuco, e Fortaleza, no Ceará, após a análise multivariada dos fatores investigados, a idade na última gestação se mostrou mais significativa no risco para a doença do que a idade na primeira gestação, mesmo controlando por esta última, pois muitas mulheres só tiveram um filho.

Também tiveram prevalência próxima de $40 \%$ a história de um ou mais abortos e o uso de contraceptivos orais por cinco anos ou mais. O estudo retrospectivo de Reis et al. 22 , realizado em instituições hospitalares das cidades de Fortaleza, Recife, Curitiba, Rio de Janeiro e Campinas, entre 1992 e 1993, com 4.408 mulheres, estimou uma prevalência de $19 \%$ de abortamento provocado, valor que, na época, já foi considerado muito alto pelos autores. A interrupção da gestação em sua fase inicial, quando o tecido mamário contém altas concentrações de estrogênios, pode favorecer a proliferação de células malignas. O discreto aumento observado no risco para a doença parece estar relacionado a alguns subgrupos de mulheres, como as nulíparas e aquelas que tiveram filhos, mas foram expostas ao fator antes da primeira gestação a termo 19 . Diferente do que acontece com outras variáveis associadas, que necessitam de exposição prolongada para que o risco da doença seja alterado, no caso do aborto basta apenas uma exposição 20 .

O estudo de Schor et al. 23, realizado em São Paulo, com 1.157 mulheres entre 10 e 49 anos, encontrou uma prevalência de $35,3 \%$ para o uso de contraceptivos orais. A revisão de Malone et al. 24 mostrou que o uso prolongado desses medicamentos aumenta o risco da doença em mulheres com menos de 45 anos. A explicação biológica para esse efeito é que os contraceptivos orais aumentam a proliferação de células epiteliais normais e também de células malignas já presentes no tecido mamário 25 . Por isso, o uso de tais medicamentos por grande número de mulheres em algumas regiões do país merece uma vigilância mais adequada quanto a um possível aumento no risco, seja para o câncer da mama, seja para outras doenças.

A prática de exercícios físicos regulares pode reduzir o risco de câncer de mama e é um fator já estabelecido como protetor por alguns autores 7,16,26. Mais da metade das mulheres da nossa amostra era sedentária, e, entre as que praticavam exercícios físicos regulares, a caminhada, que é uma modalidade de moderada intensidade, foi mais freqüente, talvez por ser mais conhecida e mais acessível. As mulheres da área rural se mostraram mais ativas, e isso pode estar relacionado ao estilo e condições de vida no campo, como trabalho na agricultura, menor oferta de transporte público e o difícil acesso ao comércio e a outros serviços básicos.

O tabagismo atual esteve presente em 17\% do grupo, um valor semelhante ao encontrado no inquérito domiciliar realizado pelo INCA 27, que foi de $16 \%$ para mulheres da cidade do Rio de Janeiro. Dados da Organização Mundial da Saúde (Tabagismo no Brasil. http://www.inca. org.br, acessado em Mai/2002) mostram que a prevalência de fumantes na área rural é maior que na área urbana, mas essa diferença não foi observada em nossos achados. Mesmo existindo uma forte ligação entre atividade estrogênica e tabagismo, a revisão de Palmer \& Rosemberg 32 mostrou que a maioria dos estudos não evidenciou uma associação significativa para o câncer de mama; entre aqueles que observaram tal associação, o aumento de risco foi de $20 \%$ a $30 \%$. Porém, como existe uma tendência de aumento da prevalência do hábito entre as mulheres, este deverá ser desencorajado em virtude de seu efeito danoso para a saúde.

O consumo excessivo de alimentos gordurosos também não tem apresentado evidência de associação positiva com o câncer de mama em algumas meta-análises 29,30. Entretanto, dados recentes e prospectivos da coorte sobre dieta e câncer, de Malmo, na Suécia, composta de 11.726 mulheres na pós-menopausa, mostram um aumento significativo no risco para a doença relacionado ao consumo alto de gorduras, e também um risco relativo de $2,12(1,2-3,6)$ relacionado ao alto consumo de vinho 31 . 
Os nossos dados mostraram baixa prevalência para este fator (7\%), quando analisada com base nas pontuações do instrumento utilizado 17, que foi construído valendo-se dos hábitos alimentares de adolescentes. Isso pode ter limitado a avaliação desse fator na nossa amostra. Embora ele integrasse uma pequena parte dos questionários utilizados pelo INCA 27 para o seu inquérito domiciliar, a versão completa publicada por este Instituto parece ser mais adequada para futuras investigações em populações semelhantes à do presente estudo.

A prevalência para a obesidade $(30 \%)$ foi pouco maior que a encontrada $(27 \%)$ no estudo transversal de Lima et al. 32 , realizado com mulheres indígenas do Mato Grosso. Esse fator de risco parece apresentar uma interação com o status menopausal das mulheres. A maioria das pesquisas mostra uma relação positiva com a doença em mulheres menopausadas e negativa em mulheres não menopausadas 33 . Contudo, é uma condição que também deve ser desencorajada em todas as mulheres, em razão de seus efeitos nocivos para a saúde, principalmente do sistema cardiovascular.

No que diz respeito às variáveis de risco mais fortemente associadas ao câncer de mama, encontramos apenas duas no nosso inquérito. Uma foi o antecedente familiar em primeiro grau, com prevalência de $3,7 \%$. Trata-se de um valor mais baixo do que o encontrado $(6,5 \%)$ no estudo transversal de Lima \& Falk 14 em mulheres nordestinas, mas muito semelhante aos encontrados entre os controles do estudo de Mendonça 15, que eram visitantes de pacientes internados em um hospital de câncer na cidade do Rio de Janeiro - 3,7\% também (quando as parentas de pacientes com câncer de mama foram retiradas da análise), e entre os controles do estudo de Hardy et al. 10, da cidade de Campinas, São Paulo, cuja prevalência foi de 3,5\%.

Acredita-se que a participação do antecedente familiar na etiologia do câncer de mama possa estar relacionada a fatores genéticos, como as mutações de BRCA1/2. Esses genes produzem proteínas que regulam o mecanismo de multiplicação celular e são conhecidos como supressores de tumores. Mutações implicam perda desse controle, e esses genes alterados são transmitidos hereditariamente. Eles podem estar presentes em cerca de $5 \%$ a $10 \%$ dos casos com a doença 7,19,34,35.

O antecedente familiar é de fácil investigação em anamneses e inquéritos, e por isso é um dos principais indicadores para o desenvolvimento de um rastreamento diferenciado nas mulheres que o apresentam. Entretanto, como é um fator de risco de baixa prevalência, e nem todas as mulheres que o apresentam irão desenvolver o câncer de mama, pouquíssimos casos serão detectados em sua fase inicial, se este for o único critério utilizado.

O segundo fator de risco mais fortemente associado ao câncer de mama encontrado neste estudo foi o antecedente pessoal da doença com uma prevalência de $0,4 \%$, muito próxima àquela de $0,5 \%$ encontrada no estudo de Lamas \& Pereira 11. Esses baixos valores podem apresentar, de forma clara, a situação do controle da doença no País, onde a maioria dos casos é diagnosticada em estágios avançados da doença, levando a uma pequena sobrevida. Por isso, é raro encontrar sobreviventes do câncer de mama entre a população geral. O mecanismo de ação desse fator de risco ainda não está totalmente esclarecido. Seu efeito parece ser maior em mulheres com antecedente familiar da doença, na pré-menopausa e/ou antecedente de carcinoma lobular in situ 7,19.

Além da limitação decorrente do uso do instrumento para avaliação da dieta, outras limitações do presente estudo merecem ser consideradas. A primeira diz respeito à população para a qual pretendíamos inferir nossos dados. Embora tenhamos uma estimativa do número de mulheres cadastradas no PSF, desconhecemos quantas delas não utilizam seus serviços e, principalmente, não sabemos se apresentam características semelhantes às daquelas que freqüentam as unidades. Portanto, a generalização dos achados desta pesquisa para a população-alvo pode estar comprometida.

Outra limitação diz respeito à seleção das entrevistadas. A intenção foi entrevistar todas as mulheres elegíveis presentes nas unidades durante os turnos de trabalho, mas algumas não foram entrevistadas porque o fluxo de atendimento foi mais rápido do que o fluxo das entrevistas. Embora este processo de "não-seleção" não tenha sofrido influência do pesquisador, não se pode descartar a introdução de um viés nas características do grupo.

Por fim, as evidências têm mostrado que um pequeno número de casos de câncer de mama pode ser explicado por variáveis associadas e que a maior parte delas não é modificável. Todavia, mesmo com suas limitações, este estudo chama a atenção para as altas prevalências de comportamentos de risco como sedentarismo, obesidade e tabagismo, cujos valores foram semelhantes aos encontrados em outros estudos. Esses fatores são importantes para os programas de saúde pública porque podem ser mudados e podem ser o tema de ações educativas para o controle não só do câncer de mama, mas também de outras doenças. 


\section{Resumo}

Um inquérito epidemiológico foi desenvolvido nas dez unidades de saúde da família do Município de Teresópolis, Rio de Janeiro, Brasil, em 2003, no qual se investigou o padrão de distribuição de variáveis associadas para o câncer de mama. Foram entrevistadas 698 mulheres entre 25 e 88 anos, 112 na área rural e 586 na urbana. Entre os fatores que apresentam forte associação com a doença, foram encontradas prevalências de $36,1 \%$ para idade de 50 anos ou mais, 3,7\% para antecedente familiar de câncer de mama em parentes de primeiro grau e 0,4\% para antecedente pessoal da doença. Para outros fatores investigados, as prevalências foram mais elevadas em história de abortos $(38,5)$, amamentação por menos de um ano (37,4\%), uso prolongado de contraceptivos orais $(41,1 \%)$, sedentarismo $(58,7 \%)$ e obesidade $(30,0)$. Embora estes últimos fatores ainda estejam em investigação e as medidas estimadas nos estudos sejam de fraca associação, eles podem ser de grande importância para a saúde pública quando suas prevalências são altas na população, no que diz respeito ao controle não só do câncer de mama, como também de outras doenças.

Fatores de Risco; Neoplasias Mamárias; Saúde da Mulher

\section{Referências}

1. Lopes ER, Rebelo MS, Abib AR, Abreu E. Câncer de mama: epidemiologia e grupos de risco. Rev Bras Cancerol 1995; 42:105-16.

2. Abreu E, Koifman. Fatores prognósticos no câncer da mama feminina. Rev Bras Cancerol 2002; 48:113-32.

3. Kligerman J. Fundamentos para uma política nacional de prevenção e controle do câncer. Rev Bras Cancerol 2002; 48:3-7.

4. Fletcher SW. Screening for breast cancer. http:// www.utdol.com/application/topic/ (acessado em Mar/2004)

5. Instituto Nacional de Câncer. Controle do câncer de mama. Documento de consenso. Rio de Janeiro: Instituição Nacional de Câncer; 2004

6. Instituto Nacional de Câncer. Programa Nacional de Controle do Câncer do Colo do Útero e de Mama - Viva Mulher. Rio de Janeiro: Instituto Nacional de Câncer 2001. http://www.inca.org.br/ (acessado em Nov/2001).

\section{Colaboradores}

V. F. S. Pinho contribuiu na realização deste inquérito, análise dos dados e redação do texto. E. S. F. Coutinho contribuiu na análise dos dados e revisão da redação do texto final.

\section{Agradecimentos}

Às Professoras Doutoras Gulnar de Azevedo e Silva Mendonça (Instituto de Medicina Social/Universidade do Estado do Rio de Janeiro) e Inês Mattos Echenique (Escola Nacional de Saúde Pública Sergio Arouca/ Fundação Oswaldo Cruz/Rio de Janeiro), pela revisão crítica da dissertação que deu origem a este artigo. À Secretaria Municipal de Saúde de Teresópolis, pela autorização da coleta de dados em suas Unidades de Saúde da Família.
7. Meister K, Morgan J. Risk factors for breast cancer. New York: American Council on Science and Health; 2000.

8. Warner E, Heisey RE, Goel V, Carrol JC , McCready DR. Hereditary breast cancer. Risk assessment of patients with a family history of breast cancer. Can Fam Physician 1999; 45:104-12.

9. Kelsey JL. Breast cancer epidemiology: summary and future directions. Epidemiol Rev 1993; 15:25863.

10. Hardy EE, Pinotti JA, Algaba MFO, Osis MJD, Faundes A. Variáveis reprodutivas e risco para câncer de mama. Estudo caso-controle desenvolvido em Campinas, São Paulo. Rev Bras Ginecol Obstet 1989; 11:212-6.

11. Lamas JM, Pereira MG. Fatores de risco para o câncer de mama e para lesões pré-malignas em mulheres assintomáticas no Distrito Federal. Rev Bras Mastol 1999; 9:108-14. 
12. Pinho VFS, Coutinho ESF. Risk factors for breast cancer: a systematic review of studies with famale samples among the general population in Brazil. Cad Saúde Pública 2005; 21:351-60.

13. Secretaria Estadual de Saúde do Estado do Rio de Janeiro. Programa Saúde da Família. Situação da estratégia da saúde da família no Estado do Rio de Janeiro - julho de 2002. http://www.saude.rj.gov. br/ (acessado em Jan/2003).

14. Lima JQ, Falk JA. A importância da identificação dos fatores de risco na luta contra o câncer. 2ạ edição. Recife: Sociedade Pernambucana de Combate ao Câncer; 1998.

15. Mendonça GAS. Pesticidas e câncer de mama - um estudo de caso-controle no Rio de Janeiro [Tese de Doutorado]. São Paulo: Universidade de São Paulo; 1997.

16. Colditz GA, Atwood KA, Emmons K, Monson RR, Willett WC, Trichopoulos D. et al. Harvard report on cancer prevention volume 4: Harvard Cancer Risk Index. Cancer Causes and Control 2000; 11: 477-88.

17. Chiara VL, Sichieri R. Food consumption of adolescents. A simplified questionnaire for evaluating cardiovascular risk. Arq Bras Cardiol 2001; 77:33741.

18. Canty L. Breast cancer risk: protective effect of an early first full-term pregnancy versus increased risk of induced abortion. Oncol Nurs Forum 1997; 24:1025-31.

19. Mahon SM. Cancer risk assessment: conceptual considerations for clinical practice. Oncol Nurs Forum 1998; 25:1535-47.

20. Brind J, Chinchilli VM, Severs WB, Summy-Long J. Induced abortion as an independent risk factor for breast cancer: a comprehensive review and meta-analysis. J Epidemiol Community Health 1996; 50:481-96.

21. Kalache A, Maguire A, Thompson SG. Age at last full-term pregnancy and risk of breast cancer. Lancet 1993; 341:33-6.

22. Reis AFF, Costa CFF, Mello CR, Almeida FML, Costa HLFF, Gabiatti JRE, et al. Estudo epidemiológico do abortamento no Brasil. Rev Bras Ginecol Obstet 1995; 17:453-61.

23. Schor N, Ferreira AF, Machado VL, França AP, Pirotta KCM, Alvarenga AT, et al. Mulher e anticoncepção: conhecimento e uso de métodos anticoncepcionais. Cad Saúde Pública 2000; 16:377-84.
24. Malone KE, Daling JR, Weiss NS. Oral contraceptives in relation to breast cancer. Epidemiol Rev 1993; 15:80-97.

25. Schlesselman JJ. Net effect of oral contraceptive use on the risk of cancer in women in the United States. Obstet Gynecol 1995; 85(Pt 1):793-801.

26. American Cancer Society. The Complete Guide - Nutriton and Physical Activity; 2001. http://www. cancer.gov/ (acessado em Mai/2002).

27. Coordenação de Prevenção e Vigilância, Instituto Nacional de Câncer. Inquérito domiciliar sobre comportamentos de risco e morbidade referida de doenças e agravos não transmissíveis. Rio de Janeiro: Secretaria de Vigilância em Saúde/Instituto Nacional de Câncer; 2004.

28. Palmer JR, Rosenberg L. Cigarette smoking and the risk of breast cancer. Epidemiol Rev 1993; 15:14556.

29. Hunter DJ, Spiegelman D, Adami H, Beeson WL, Brandt PA, Folsom AR et al. Cohort studies of fat intake and the risk of breast cancer - a pooled analysis. N Engl J Med 1996; 334:356-61.

30. Missmer SA, Smith-Warner SA, Spiegelman D, Yaun S, Adami H, Beeson WL et al. Meat and dairy food consumption and breast cancer: a pooled analysis of cohort studies. Int J Epidemiol 2002; 31:78-85.

31. Mattisson I, Wirfät E, Wallströn P, Gullberg B, Olsson H, Berglund G. High fat and alcohol intakes are risk factors of post-menopausal breast cancer: a prospective study from the Malmö diet and cancer cohort. Int J Cancer 2004; 110:589-97.

32. Lima MG, Koifman S, Scapulatempo IL, Peixoto M, Naomi S, Amaral MC. Fatores de risco para câncer de mama em mulheres indígenas Teréna de área rural, Estado do Mato Grosso do Sul, Brasil. Cad Saúde Pública 2001; 17:1537-44.

33. Hunter DJ, Willett WC. Diet, body size, and breast cancer. Epidemiol Rev 1993; 15:110-31.

34. Koifman RJ, Koifman S, Vieira RJS. Familial aggregation of breast/ovarian cancer: age of onset along subsequent generations in Brazil. Cad Saúde Pública 1998; 14:181-5.

35. National Cancer Institute. Genetics of breast and ovarian cancer. Cancer genetics - health professionals; 1999. http://www.nci.nih.gov/ (acessado em Fev/2000).

Recebido em 08/Nov/2005

Versão final reapresentada em 29/Jun/2006

Aprovado em 28/Nov/2006 